\title{
Integrated visualization of multi-angle bioluminescence imaging and micro CT
}

\author{
P.Kok ${ }^{\mathrm{a}, \mathrm{b}}$, J. Dijkstra ${ }^{\mathrm{a}}$, C.P. Botha ${ }^{\mathrm{a}, \mathrm{b}}$, F.H. Post ${ }^{\mathrm{b}}$, E. Kaijzel $^{\mathrm{c}}$, I. Que ${ }^{\mathrm{c}}$, C.W.G.M. Löwik ${ }^{\mathrm{c}}$, \\ J.H.C. Reiber ${ }^{\mathrm{a}}$, B.P.F. Lelieveldt $\mathrm{t}^{\mathrm{a}}$, \\ ${ }^{a}$ Division of Image Processing, Leiden University Medical Center, Leiden, the Netherlands \\ ${ }^{\mathrm{b}}$ Visualization Group, Delft University of Technology, Delft, the Netherlands \\ ${ }^{c}$ Dept of Endocrinology, Leiden University Medical Center, Leiden, the Netherlands
}

\begin{abstract}
This paper explores new methods to visualize and fuse multi-2D bioluminescence imaging (BLI) data with structural imaging modalities such as micro CT and MR. A geometric, back-projection-based 3D reconstruction for superficial lesions from multi-2D BLI data is presented, enabling a coarse estimate of the 3D source envelopes from the multi-2D BLI data. Also, an intuitive 3D landmark selection is developed to enable fast BLI / CT registration. Three modes of fused BLI / CT visualization were developed: slice visualization, carousel visualization and 3D surface visualization. The added value of the fused visualization is demonstrated in three small-animal experiments, where the sensitivity of BLI to detect cell clusters is combined with anatomical detail from micro-CT imaging.
\end{abstract}

Keywords: Bioluminescence imaging, Micro CT, small animal imaging, image fusion, registration, data visualization

\section{INTRODUCTION}

Bioluminescence imaging (BLI) is a relatively novel imaging technique that has found widespread application in lifesciences research over the past decade [1]. BLI is based on a light-emitting chemical reaction that occurs in nature in bioluminescent animals such as fireflies and some algae and jellyfish species. For instance, in fireflies, the enzyme luciferase catalyzes the conversion of a substrate luciferin into oxiluciferin, which emits photons during the process. By integrating the luciferase encoding gene into living cells, luciferase will be produced in the cell. After adding luciferin, this same bioluminescence reaction can be induced in this cell, causing it to emit light. The production of luciferase can be made switchable, such that luciferase only is produced when a particular gene is activated. This selective activation has enabled a range of possibilities to track cells and monitor the function of specific genes and processes in the cellular biochemistry with a high sensitivity; this all in the living animal.

To visualize the BLI activity, the anaesthetized animal is placed in a dark acquisition chamber, and using a sensitive, cooled CCD camera, the bioluminescent signal can be detected. Data is typically acquired in conjunction with a photograph in visible light taken with the same camera (see Figure 1). Most commercially available systems enable 2D imaging. However, more recently, optical molecular imaging instrumentation is advancing towards 3D imaging. This is realized by acquiring emission and visible light images from several angles. This provides information about the 3D position of the light source in the animal (see Figure 2).

The aforementioned 2D and 3D BLI also have a few limitations: they are highly sensitive in detecting very small cell clusters, long before any structural changes become apparent; however they lack anatomical detail, and are mainly semiquantitative. Structural modalities such as MR and CT on the other hand, have a low sensitivity to small masses, but high spatial resolution, enabling better lesion shape and size characterization. The combination of optical and structural imaging would enable a much more sensitive and accurate source localization and quantification, amplifying the utility of the individual modalities.

*b.lelieveldt@lumc.nl

Medical Imaging 2007: Visualization and Image-Guided Procedures, edited by Kevin R. Cleary, Michael I. Miga, Proc. of SPIE Vol. 6509, 65091U, (2007) · 1605-7422/07/\$18 · doi: 10.1117/12.711117 

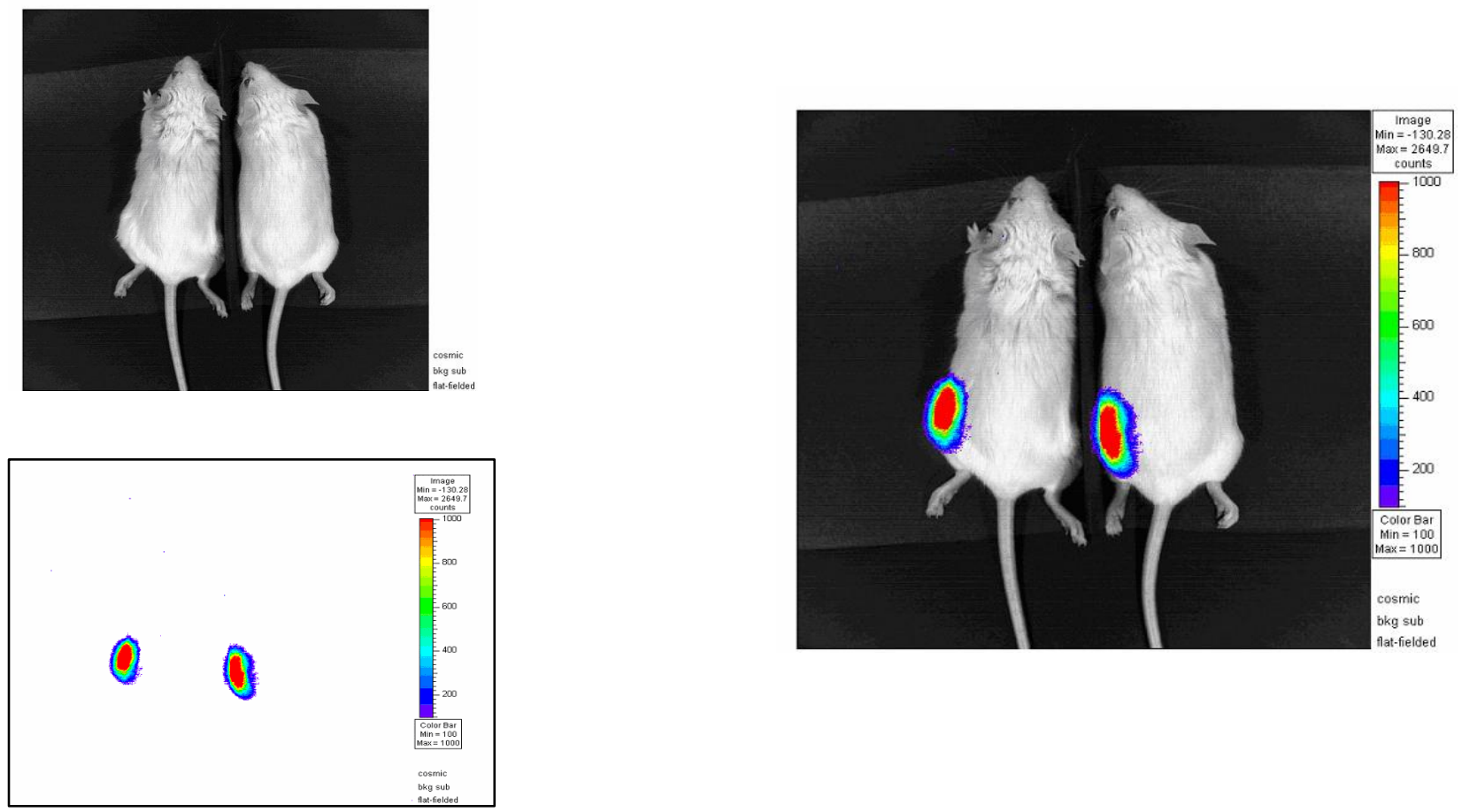

Figure 1: Biolominescence images are usually acquired in pairs: a visible light photograph (top left) and a photon emission image (bottom left), which is typically viewed as an overlay on the visible light image (right image).
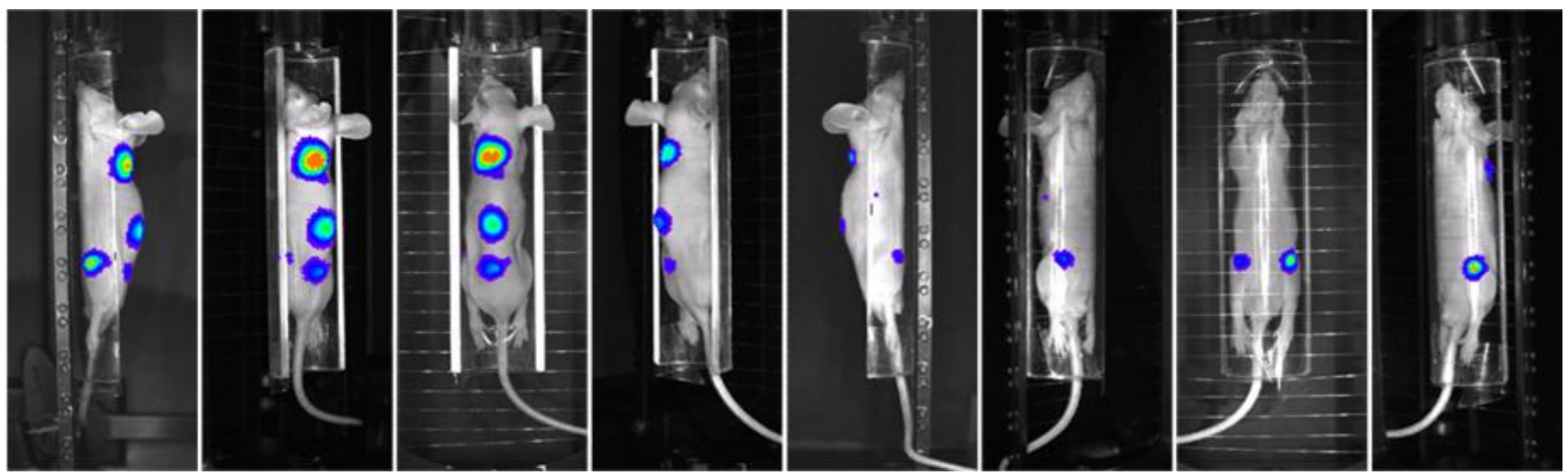

Figure 2: Multi-view bioluminescence imaging, where images are acquired sequentially in 45 degree angles surrounding the subject.

The goal of this work was to develop tools for an effective and intuitive exploration of fused multi-angle BLI / micro CT data. In this paper, we introduce the following novel elements:

- An algorithm to make a rough geometric approximation of the 3D light source location based on a number of multi-angle BLI images. This source approximation serves to highlight potential structural abnormalites in the structural imaging data.

- An intuitive 3D landmark selection to enable fast multi-view BLI / CT registration

- Three modes of interactive visualization to enable an intuitive exploration of the fused data.

The efficacy of the fused visualization is demonstrated in three small-animal case studies, where the sensitivity of BLI to detect small cell clusters is combined with anatomical detail from micro-CT. 


\section{METHODS}

We developed a modular visualization platform called INTERGRIM (INTEGRated IMaging) that allows fast implementation and testing of visualization algorithms for fusion of structural imaging (MR and CT) and optical molecular imaging data. It has been designed as a pipeline-type architecture, where three separate components can be distinguished. The first deals with the coarse geometry-based reconstruction of superficial 3D BLI hotspots from multiangle 2D BLI images. The second focuses on registration of data sets between different modalities. The third part consists of fusion and visualization of the data from multiple modalities. Below, we describe three components that were implemented specifically for the problem of fusing multi-angle 2D BLI images with CT data.

\subsection{Geometric approximation of the 3D light source location}

Currently available multi-angle BLI scanners typically acquire a fixed number of views surrounding the subject. The transformations corresponding to the views are known and consist of a rotation about one axis and a projection onto the image plane. Based on the known geometry of the imaging system, we developed an elementary geometric reconstruction, where the BLI signal is back-projected into a reconstruction volume. For each voxel in the reconstruction volume, the corresponding intensity value is retrieved for each of the $\mathrm{N} 2 \mathrm{D}$ views. The current voxel is assigned the average intensity over all views:

$$
V\left(p_{x, y, z}\right)=\sum_{i}^{N} \frac{I_{i}\left(T_{i}\left(p_{x, y, z}\right)\right)}{n}
$$

where $V(p)$ is the intensity at the specified point in the volume, $I(p)$ is the intensity at the corresponding point in the $2 \mathrm{D}$ images, $T$ represents the projection transformations and $n$ is the number of projections.

It should be noted that this approach does not represent an exact tomographic signal strength reconstruction: we aim to approximate the $3 \mathrm{D}$ location of the light source in order to a direct the attention of the user to possible areas of interest in the structural imaging modality. We do not correct for absorption, scattering or refraction, as a result the application of our method is mainly limited to superficial lesions. Methods for accurate bioluminescence tomography of deeper BLI sources require non-linear photon models, which have been reported elsewhere [2-9]; as yet, heterogeneous tissue models have proven to be computationally very expensive, and only using simplified assumptions about optical tissue properties, some of these methods can be deployed for on-line, interactive application [4].

\subsection{Landmark-based registration of BLI and CT}

Although BLI in itself does not contain any structural information that can be used for registration, each BLI acquisition consists of a visible-light photograph and a corresponding BLI emission image. Because these photographs have been acquired with the same camera, these images are expressed in the same coordinate space, and can therefore be used to register the BLI images with structural modalities such as CT. Based on this, we developed a landmark based registration method as outlined in Figure 3.

An interactive user interface was developed to identify corresponding anatomical landmark pairs in the BLI and CT data as follows. To obtain a 3D landmark from the 2D BLI photographs, the user is required to indicate two points in two separate photographs that are not opposite from each other, representing the same characteristic anatomical landmark. For this purpose, a line is back projected into the volume for both points. The 3D location of the landmark is defined as the intersection point of the projection lines, where the distance to both lines is equal and minimal. The third point is directly selected in the $\mathrm{CT}$ volume on the corresponding location using orthogonal slice viewing. Thus, three points are required for one landmark pair. A minimum of three non co-planar landmarks is required to register both data sets. 


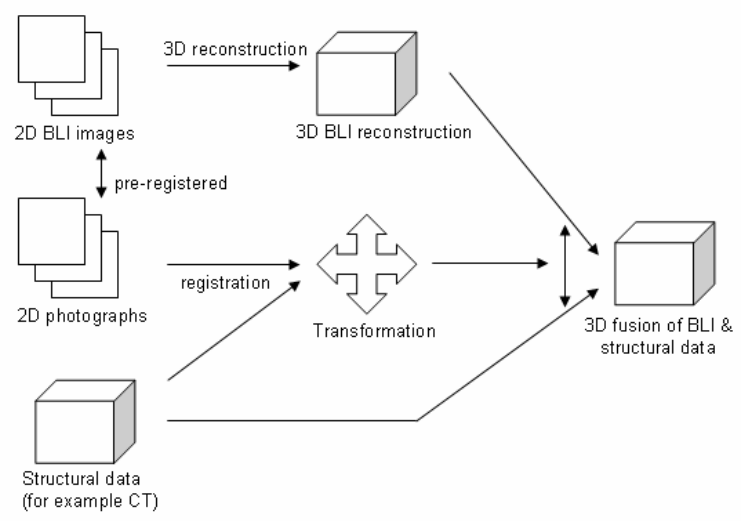

Figure 3 Overview of the registration process. The structural modality is registered with the BLI data using the visible light images. The resulting transformation is used to fuse the structural modality with the 3D reconstruction of the emission BLI data

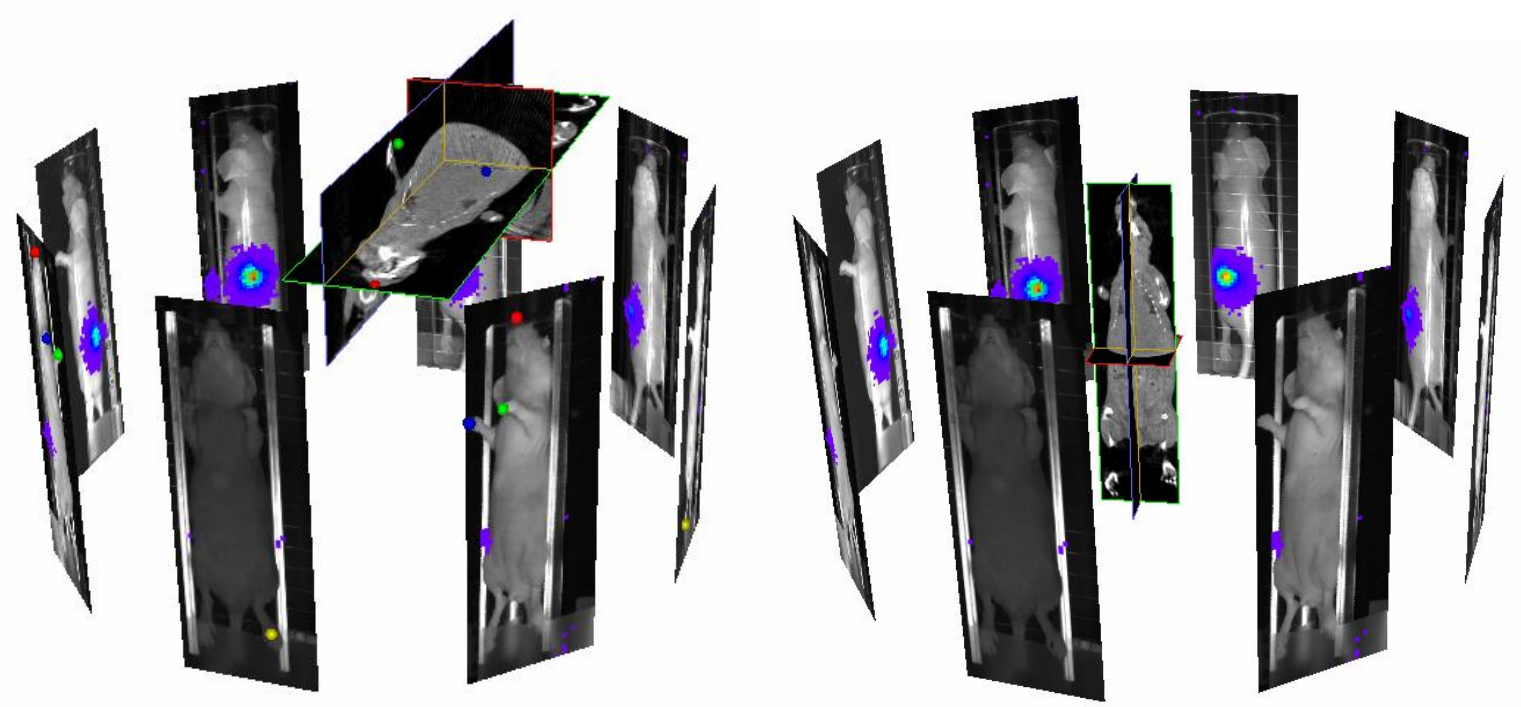

Figure 4 Interactive manual landmarking was developed to enable a landmark-based rigid registration. The user indicates 3 characteristic anatomical landmarks in two non-opposing BLI views, and the same landmarks in the CT data (colored balls in the left pane). Based on these corresponding landmarks, the micro CT data is rigidly registered to the multi-view BLI data.

\subsection{Combined visualization of BLI and CT}

After registration, the BLI and CT data can be jointly visualized. We explored three visualization modes, which provide an intuitive exploration of the BLI source location, the relation between the 2D projections and the 3D reconstruction and a fused visualization of the BLI and CT data:

Fused slice visualization consists of an orthogonal slice browser for the CT data, with color enhanced BLI sources. The structural data is encoded as intensity values in the HSI signal, while the BLI data is represented by the hue. In addition, semi-transparent iso-surfaces around the light sources are generated using Marching Cubes [10]. The iso-threshold for these surfaces is manually chosen. This results in a visualization where the reconstructed light sources can be easily localized using the surfaces. In addition, the structural environment of the light source can be further inspected using the color-coded data representation. 
Carousel visualization: the multi-2D BLI data is visualized and linked in the 3D space in an intuitive manner by placing the 2D images around the 3D CT volume and the reconstructed light sources. This visualization is interactive: if a point on one of the 2D images is clicked, the corresponding point in the 3D data is connected to it with a line. If the 3D surface reconstruction is clicked on, the point is projected onto each 2D image and connected with a line. Physically placing the 2D images around the 3D reconstruction and explicitly visualizing the relationship between points in 3D space and their 2D views makes this technique useful, especially for users that are accustomed to conventional 2D image inspection.

Volume-surface visualization shows a surface reconstruction of the BLI sources together with a volume visualization of the CT data. Also here, the BLI source surfaces are derived using Marching Cubes [10] with a manually chosen isothreshold. This provides a quick overview of the approximate location of the light sources within the subject.

\section{EXPERIMENTAL SETUP}

To test the developed platform, we performed a pilot evaluation study on small animal imaging data that was acquired within ongoing experimental protocols at our institution. A qualitative validation on three case studies was performed to evaluate two aspects:

1) Correctness of the $3 D$ reconstructed source location. We selected data from two experiments in mice, where bioluminescent cells were injected at a known anatomical location, as to visually verify the reconstructed BLI sources with the injection site as seen in the micro CT data. In subject 1, 100000 RC21-luc cells, a luciferase expressing human renal carcinoma cell line, were injected under the renal capsule. Four weeks after implantation, a luc-expressing renal cell carcinoma was established and the mouse was scanned. Subject 2 was injected with $100 \mu 1100000 \mathrm{KS}-H i s L u c$ cells, a human luciferase-expressing stem cell line, into the left heart ventricle three weeks before the scan.

2) Complementarity of BLI (sensitivity) and micro CT (structural detail). We explored this aspect by studying the interaction between breast cancer metastases and the skeleton, where BLI provides a sensitive source detection and CT provides a detailed monitoring of bone resorption in the skeleton. Breast cancer has a preference for bone to metastasize, and at the location of a metastatic lesion, the bone is broken down and resorbed, causing structural damage in the skeleton, such as fractures or completely resorbed bones. To this end, we selected a study, where subject 3 was injected with luciferase positive human MDA231 breast cancer cells into the cardiac left ventricle. The animal was scanned 40 days after cell injection to screen for possible metastases.

In all experiments, the following imaging protocol was applied: five minutes before the scan, luciferin was injected,and the mouse was then recorded for 30 seconds per image with a Xenogen VIVO Vision IVIS 3D scanner (Alameda, CA, USA) at a range of wavelengths between 585 and $700 \mathrm{~nm}$. Micro CT-scans were acquired with a SkyScan 1178 microCT scanner (Aartselaar, Belgium) at a resolution of $80 \times 80 \times 80 \mu \mathrm{m}$. The acquired scans were subsequently reconstructed, registered and visualized using the INTEGRIM platform, and qualitatively assessed by an expert observer.

\section{RESULTS}

The resulting visualizations are presented in Figures 5,6 and 7. For subject 1, a strong signal at the location of the kidney was expected, since the tumor cells were injected directly under the renal capsule. Figure 5(a) and 5(c) show that there is one light source present near the spine of the mouse. A closer inspection of the color-coded fusion in Fig. 5(a) shows in more detail that the light source is indeed located in the kidney. The added value of Fig. 5(b) is that it shows the orientation of the $2 \mathrm{D}$ projections around the reconstruction volume as well as the explicit relation between the $2 \mathrm{D}$ and 3D BLI data. For subject 2 (Figure 6), a strong signal in the cardiac area of the chest was expected at the site of the stem cell injection. As in subject 1, there is a clear correspondence between the expected location and the reconstructed source location, where one source in visible in the cardiac area of the chest.

For subject 3, it was expected that the locations of strong signals in the BLI data were located near, in or on the skeletal bones, and that structural changes take place in the bone structures due to bone destruction. Here, no signal was expected at the cell injection site (the cardiac left ventricle), but more downstream in the circulation and in the skeletal structures. Several lesions in or on the skeletal structures were detected. Also, the combined visualization clearly reveals the bone resorption in the $\mathrm{CT}$ at the location of the osteolitic metastases as identified in the multi-angle BLI. 


\section{DISCUSSION}

This paper presents a method to approximate the light source location from multi-angle 2D BLI images, a method to register BLI and structural data and three new visualization modes for fusion of BLI and structural imaging data. We applied these techniques to three case studies and presented the resulting visualizations: the correctness of the approximated BLI source location was verified by performing two experiments where cells injected at a known location. In both experiments, results correlated with our expectations about the natural behavior of the injected cells, and the reconstructed BLI hotspots corresponded to the cell injection sites. Second, the complementarity of BLI for detection sensitivity and CT for anatomical detail was demonstrated in one case study, where we found that 1) only BLI hotspots were reconstructed in close proximity of the skeletal structures, and 2) at these skeleton-based BLI hotspots, clear bone resorption and destruction was visible in the CT data. These experiments underscored the added value of the fused visualization compared to the traditional 2D BLI analysis workflow. Further quantitative evaluation in phantom experiments and in a larger set of small animal experiments is ongoing.

There are a few inherent limitations to the presented work. The back-projection based reconstruction method was designed to approximate the location of the light source position. It does not take into account non-linear photon propagation effects such as absorption, scattering and refraction. As a result, a positioning error may occur, that increases with the thickness and composition of the tissue layer. In our case, the main interest was to detect superficial lesions, in which case this positioning error is expected to be less pronounced, which is confirmed by the experiments. However, using a structural representation such as a pre-segmented micro CT set, tissue specific scattering, absorption and refraction parameters be assigned to the different tissues, and non-linear photon behavior can be modeled and corrected for. This way, a more accurate estimate of the location, and a quantification of the signal intensity of the light sources can be achieved, as has been shown by [2-9]. This is a necessary step for restoring the proportionality of the signal to the number of light emitting cells, which is lost due to the non-linear photon behavior in the tissues.

Another limitation of the presented method is the manual registration step, which requires user interaction in the form of landmark placement. Also, this method assumes a rigid-body registration, and therefore cannot accommodate for variations in articulation positioning between scans, either inter-modality or in follow-up scans. Therefore, we are currently developing an atlas-based articulated registration method based on matching an anatomical atlas to the micro CT data [11]. Subsequent registration of this model to the animal silhouettes as seen in the multiple BLI visible light photographs will enable an automated registration of BLI and CT data in follow-up studies that accounts for postural differences between scans.

\section{ACKNOWLEDGEMENTS}

The authors gratefully acknowledge Dave Panzarella and Kevin Francis from Xenogen / Caliper for providing the Xenogen IVIS 3D BLI camera system used in this research, and Elke van de Casteele from Skyscan for providing the Skyscan micro CT scanner used in this research. 

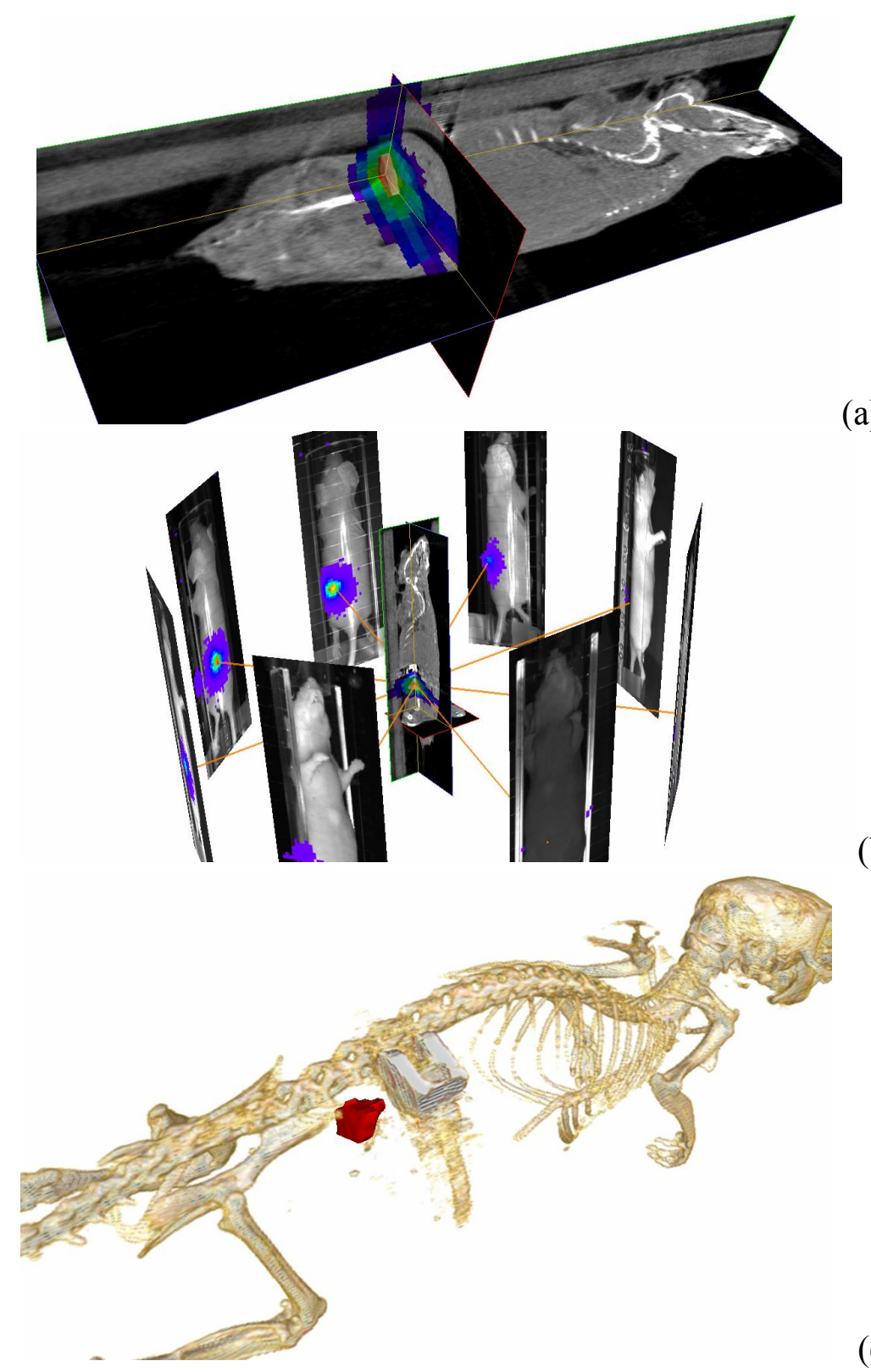

(b)

(c)

Figure 5 Visualization for subject 1, where luminescent cells were injected under the renal capsule.

(a) slice visualization of the CT data that has been fused with the reconstructed BLI data. A color map is applied to the reconstruction function (eq. 1) to indicate the light source location. Also, an iso-surface is displayed at this location.

(b) shows the explicit correspondence between a point in the 3D BLI reconstruction and the original 2D BLI views.

(c) shows a volume visualization with an iso-surface to indicate the location of the light source. Note the clamp above and to the right of the light source that was attached after surgery. These figures show that the light source is indeed located in the area of the kidney, where the luminescent cells were injected. 

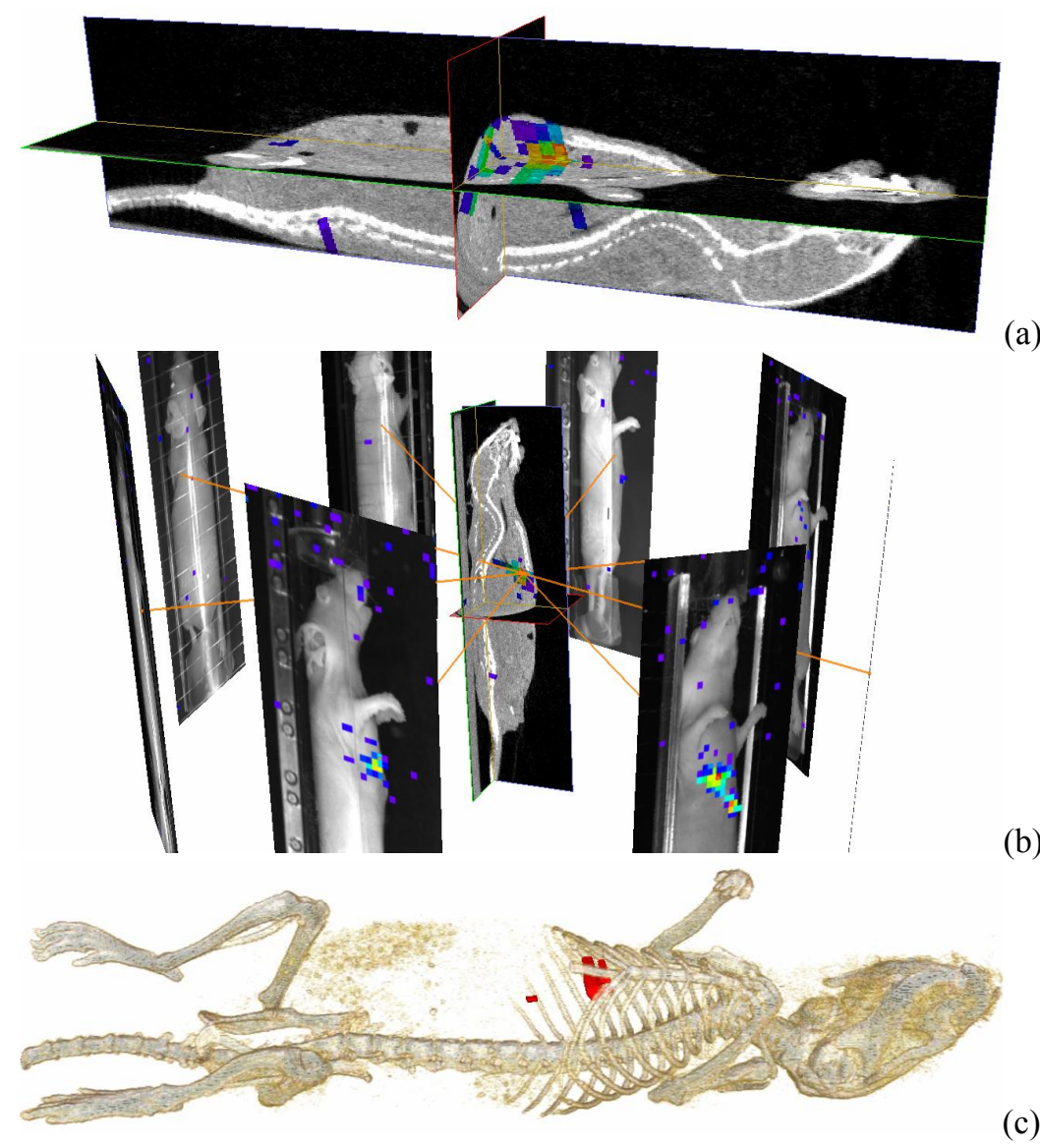

(a)

(b)

(c)

Figure 6: Fused visualizations of subject 2, where luminescent stem cells were injected in the heart, showing the slice, carrousel and volume visualization modes in a,b,c respectively, which give a good impression of the location stem cell injection 

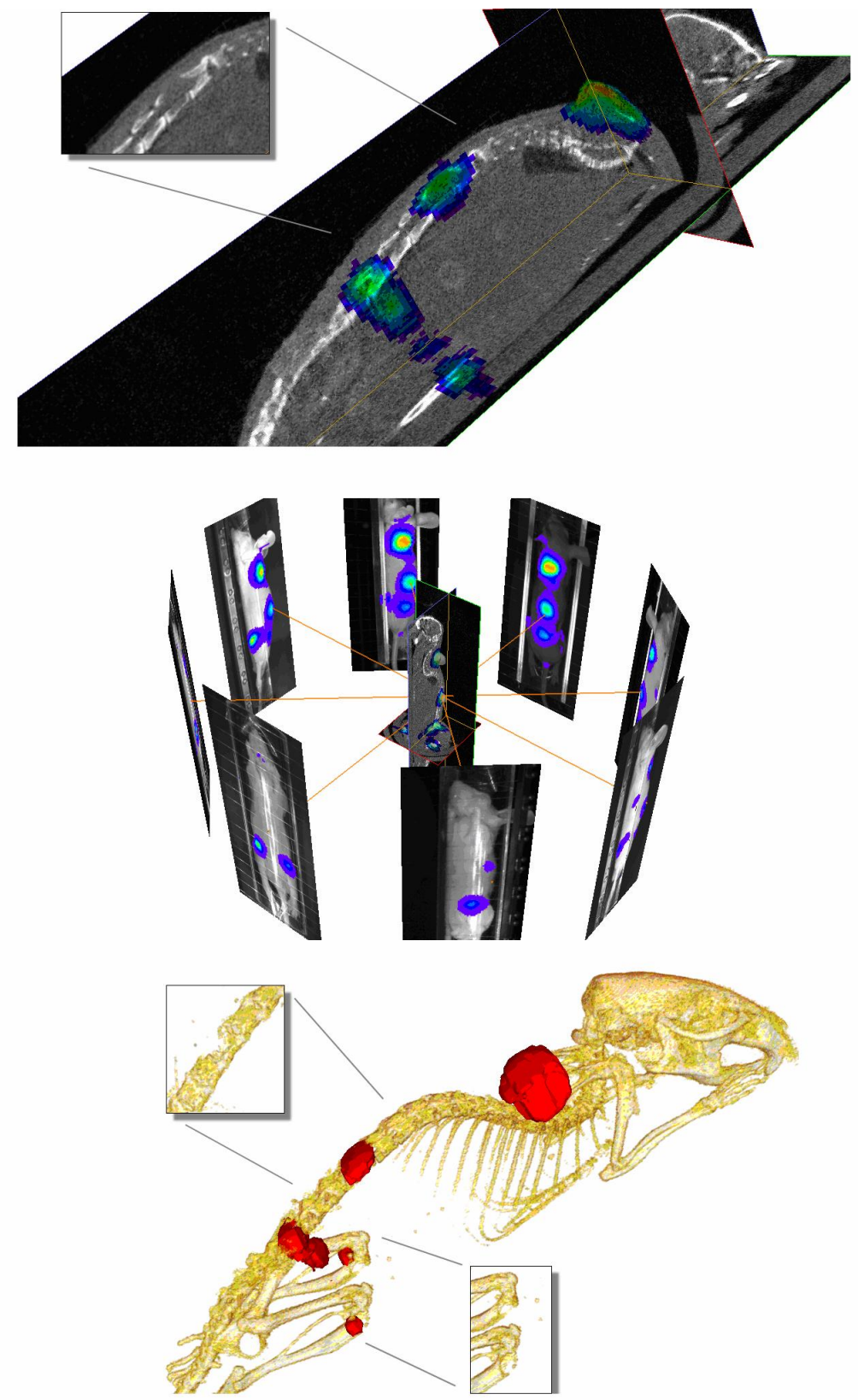

Figure 7: Fused visualizations of subject 3, where breast cancer cells were injected into the circulation, showing the slice, caroussel and volume visualization modes in a,b,c respectively. The cutouts in a and c clearly show bone destruction at the location of the metastatic breast cancer lesions in the CT data, whereas the BLI sources (in red) highlight the potential lesion locations. 


\section{REFERENCES}

1. Ntziachristos V, Ripoll J, Wang LV, Weissleder R. "Looking and listening to light: the evolution of whole-body photonic imaging." Nat Biotechnol. 2005 Mar;23(3):313-20.

2. Wang, G., Li, Y., and Jiang, M., "Uniqueness theorems in bioluminescence tomography," Medical Physics, vol. 31(8), pp. 2289-2299, 2004.

3. Troy, R., Coquoz, O., Kuo, C., Zwarg, D., Rice, B. Single-View Bioluminescent Tomography in Small Animal Models, proc. SMI 2005, abstract 513

4. Kuo, C., Coquoz, O., Troy, T., Zwarg, D., Rice, Bioluminescent Tomography for in vivo Localization and Quantification of Luminescent Sources from a Multiple-view Imaging System, proc. SMI 2005, abstract 513

5. Alexandrakis, G., Rannou, F. R., and Chatziioannou, A. F., "Effect of optical property estimation accuracy on tomographic bioluminescence imaging: simulation of a combined optical-PET (OPET) system," Physics in Medicine and Biology, vol. 51(8), pp. 2045-2053, 2006.

6. Alexandrakis, G., Rannou, F. R., and Chatziioannou, A. F., "Tomographic bioluminescence imaging by use of a combined optical-PET (OPET) system: a computer simulation feasibility study," Physics in Medicine and Biology, vol. 50( 17), pp. 4225-4241, 2005.

7. Cong, W. X., Durairaj, K., Wang, L. V., and Wang, G., "A Born-type approximation method for bioluminescence tomography," Medical Physics, vol. 33(3), pp. 679-686, 2006.

8. Cong, W. X., Wang, G., Kumar, D., Liu, Y., Jiang, M., Wang, L. V., Hoffman, E. A., McLennan, G., McCray, P. B., Zabner, J., and Cong, A., "Practical reconstruction method for bioluminescence tomography," Optics Express, vol. 13(18), pp. 6756-6771, 2005.

9. Li, H., Tian, J., Zhu, F. P., Cong, W. X., Wang, L. V., Hoffman, E. A., and Wang, G., "A mouse optical simulation environment (MOSE) to investigate bioluminescent phenomena in the living mouse with the Monte Carlo method," Academic Radiology, vol. 11(9), pp. 1029-1038, 2004.

10. W. Lorensen, H. Cline "Marching Cubes: a high resolution 3D surface constructionalgorithm", Proc. of ACM SIGGRAPH, 1987, pp. 163-169, Association for Computing Machinery

11. M. Baiker, J. Milles, A.M. Vossepoel, I. Que, E.L. Kaijzel, C.W.G.M. Löwik, J.H.C. Reiber, J.Dijkstra, B.P.F. Lelieveldt, "Fully automated whole-body registration in mice using an articulated skeleton atlas", paper nr 1430, proc. ISBI 2007, in press 2007 